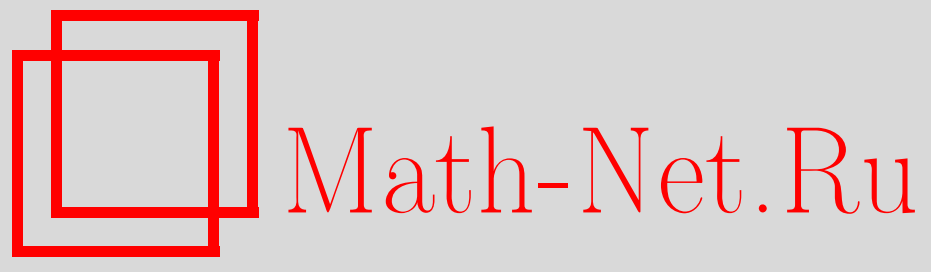

А. Торгашев, М. Петрович, О спектре оператора Лапласа бесконечного графа, Матем. заметки, 2006, том 80, выпуск 5, 773-785

DOI: https://doi.org/10.4213/mzm3087

Использование Общероссийского математического портала Math-Net.Ru подразумевает, что вы прочитали и согласны с пользовательским соглашением http://www .mathnet.ru/rus/agreement

Параметры загрузки:

IP : 34.227 .88 .159

26 апреля 2023 г., 05:27:37

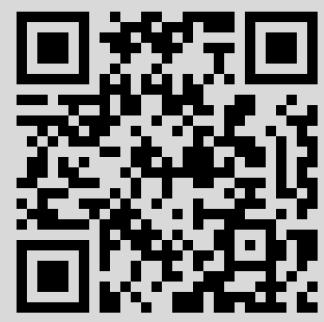




\section{О СПЕКТРЕ ОПЕРАТОРА ЛАПЛАСА БЕСКОНЕЧНОГО ГРАФА}

\section{А. Торгашев, М. Петрович}

В этой статье мы вводим понятие спектра оператора Лапласа на бесконечном счетном графе; наш способ отличен от принятого Мохаром. Мы доказываем некоторые основные свойства введенного таким образом спектра. Принятый нами подход близок к нашему подходу к определению предельного спектра бесконечного графа. В этом подходе мы существенно используем технику спектра оператора Лапласа конечных графов.

Библиография: 12 названий.

В этой статье мы вводим понятие спектра оператора Лапласа на бесконечном счетном графе; наш способ отличен от принятого Мохаром [1]-[4] и т.д. Мы доказываем некоторые основные свойства введенного таким образом спектра. Принятый нами подход близок к нашему подходу к определению предельного спектра бесконечного графа (см., например, [5], [6] или книгу [7], а также [8], [9]). В этом подходе мы существенно используем технику спектра оператора Лапласа конечных графов.

1. Введение. Начнем с краткого напоминания некоторых элементарных сведений о спектре оператора Лапласа конечного графа, которые нам понадобятся в дальнейшем. Их доказательства могут быть найдены в [10], [7] или [11], [12]. Пусть $G$ - простой граф на $n$ вершинах, $V(G)=\left\{v_{1}, \ldots, v_{n}\right\}$. Пусть $A(G)=\left[a_{i j}\right]-$ его $(0,1)$-матрица примыканий, и пусть $D(G)=\operatorname{diag}\left(d_{1}, \ldots, d_{n}\right)$ - диагональная матрица, диагональные элементы которой - это валентности $d_{1}, \ldots, d_{n}$ вершин $v_{1}, \ldots, v_{n}$. Матрица $L(G)=D(G)-A(G)$ называется матрицей Лапласа графа $G$. Она симметрична, вырожденна и положительно полуопределена. Все ее собственные значения вещественны и неотрицательны; они и образуют спектр оператора Лапласа $\sigma_{L}(G)=$ $\left\{\lambda_{1}, \ldots, \lambda_{n}\right\}$ графа $G$. Ниже мы всегда предполагаем, что $\lambda_{1} \geqslant \lambda_{2} \geqslant \cdots \geqslant \lambda_{n}$. Хорошо известно, что $\lambda_{n}=0$, причем кратность собственного значения 0 совпадает с числом компонент связности графа $G$.

Теорема А. Справедливо неравенство $\lambda_{1}(G) \leqslant n$.

ТЕОРема В. Если в графе $G$ есть хотя бы одно ребро и $d=\max \left\{d_{1}, \ldots, d_{n}\right\}$, то $d+1 \leqslant \lambda_{1}(G) \leqslant 2 d$.

ТеОРема С. Если $H$ - (не обязателъно индуцированный) подграф конечного граga $G, m o$

$$
\lambda_{k}(H) \leqslant \lambda_{k}(G), \quad i=1, \ldots,|H| .
$$

(C) А. Торгашев, М. Петрович, 2006 
Пусть теперь $G_{1}=\left(V\left(G_{1}\right), E\left(G_{1}\right)\right)$ и $G_{2}=\left(V\left(G_{2}\right), E\left(G_{2}\right)\right)$ - два конечных графа с непересекающимися множествами вершин $V\left(G_{1}\right)$ и $V\left(G_{2}\right)$ соответственно. Прямая сумма $G=G_{1}+G_{2}$ этих графов - это граф с множеством вершин $V(G)=V\left(G_{1}\right) \cup$ $V\left(G_{2}\right)$ и множеством ребер $E(G)=E\left(G_{1}\right) \cup E\left(G_{2}\right)$.

Tеорема D. Для прямой суммы $G=G_{1} \dot{+} G_{2}$ графов $G_{1}, G_{2}$ выполняется равенство

$$
\sigma_{L}\left(G_{1}+G_{2}\right)=\sigma_{L}\left(G_{1}\right) \cup \sigma_{L}\left(G_{2}\right)
$$

с учетом кратностей.

Теорема Е. Для дополнительного графа $\bar{G} \kappa$ конечному графу $G$ выполняется равенство

$$
\lambda_{k}(\bar{G})=n-\lambda_{n-k}(G), \quad k=1, \ldots, n-1 .
$$

2. Основные определения и результаты. Пусть $G$ - произвольный простой бесконечный счетный граф с множеством вершин $V(G)=\left\{v_{1}, v_{2}, \ldots\right\}$ и множеством ребер $E(G)$. Отметим, что мы не накладываем никаких ограничений на множество ребер $E(G)$, допуская, тем самым, пустое множество ребер, т.е. мы включаем в рассмотрение бесконечный граф $E_{\infty}$, состоящий лишь из изолированных вершин, и полный граф $K_{\infty}$. В частности, мы не предполагаем, что валентности вершин конечны или равномерно ограничены. Если $H$ - конечный или бесконечный (не обязательно индуцированный) подграф бесконечного графа $G$, то мы используем запись $H \subseteq G$. Последовательность конечных индуцированных подграфов $G_{n}, n \in \mathbb{N}$, бесконечного графа $G$ называется базисной для $G$, если для всякого $n$ граф $G_{n}$ является индуцированным подграфом графа $G_{n+1}$ и $\bigcup_{n=1}^{\infty} V\left(G_{n}\right)=V(G)$. Очевидно, что базисная последовательность не единственна, но в каждом графе существует, по крайней мере, одна такая последовательность. Если, кроме того, граф $G$ связен, то легко доказать, что в нем есть, по крайней мере, одна базисная последовательность, также состоящая из связных графов. Пусть теперь граф $G$ бесконечен и пусть $G_{n}$, $n \in \mathbb{N},-$ произвольная базисная последовательность индуцированных подграфов в нем. Определим $k$-е положительное собственное значение оператора Лапласа $\lambda_{k}(G)$ графа $G$ при натуральном $k$ равенством

$$
\lambda_{k}(G)=\lim _{n \rightarrow \infty} \lambda_{k}\left(G_{n}\right) \leqslant+\infty,
$$

если $\lambda_{k}\left(G_{n}\right)>0$ для хотя бы одного натурального числа $n$. Если $\lambda_{k}\left(G_{n_{0}}\right)>0$ для некоторого $n_{0} \in \mathbb{N}$, то по теореме $\mathrm{C}$ числа $\lambda_{k}\left(G_{n}\right), n \geqslant n_{0}$, образуют возрастающую последовательность вещественных чисел, поэтому указанный выше предел всегда существует и либо является конечным положительным числом, либо равен $+\infty$. Кроме того, если $\lambda_{k}(G)$ существует, то

$$
\lambda_{k}(G)=\sup _{n \geqslant n_{0}} \lambda_{k}\left(G_{n}\right)
$$

Может оказаться и так, что положительных собственных значений оператора Лапласа $\lambda_{k}(G)$ нет; это так, например, для $G=E_{\infty}$. По определению

$$
\lambda_{1}(G) \geqslant \lambda_{2}(G) \geqslant \cdots>0,
$$


однако эта последовательность может быть либо конечной (и даже пустой), либо бесконечной. Мы называем эту последовательность положителъной частъю спекmpa оператора Лапласа графа $G$ и обозначаем через $S(G)$. По аналогии с конечным случаем обозначаем через $m_{G}(\lambda)$ кратность собственного значения оператора Лаnласа $\lambda \in S(G)$, определяемую как число вхождений этого собственного значения в последовательность (1). Таким образом, $m_{G}(\lambda)$ либо конечно, либо равняется $\infty$. Полный спектр оператора Лапласа $\sigma_{L} T(G)$ определяется теперь как результат добавления к последовательности (1) собственного значения $\lambda_{0}(G)=0$ с кратностью $m_{G}(0)$, равной числу компонент связности графа $G$. Таким образом, либо $m_{G}(0)=1,2, \ldots$, либо $m_{G}(0)=\infty=\aleph_{0}$. Очевидно, тем самым, что $m_{G}(0)=1$ в том и только том случае, если граф $G$ связен. Значит, по определению

$$
\sigma_{L}(G)=\left\{\lambda_{1}(G), \lambda_{2}(G), \ldots ; 0,0, \ldots\right\} .
$$

Для конечного или бесконечного графа $G$ мы обычно обозначаем положительную часть спектра его оператора Лапласа через $S(G)$. Говоря о спектре оператора Лапласа бесконечного графа, мы обычно имеем в виду лишь его положительную часть $S(G)$. Отметим, что приведенное выше определение спектра оператора Лапласа применимо и к конечным графам, и легко видеть, что в этом случае оно дает обычный спектр оператора Лапласа конечного графа. Таким образом, наше определение естественно распространяет понятие спектра оператора Лапласа конечного графа на бесконечный случай. Приводимое ниже предложение также показывает, насколько близки эти два случая. Для конечного графа $F$ бесконечный граф $G=F \dot{+} E_{\infty}$ можно назвать бесконечным расширением графа $F$.

ПреДЛОЖЕНИЕ 1. Для бесконечного расширения $G=F+E_{\infty}$ конечного графа $F$ справедливо равенство $S(G)=S(F)$.

ДокАЗАтельство. Последовательность конечных графов $G_{n}=F \dot{+} E_{n}, n \in \mathbb{N}$, в $G$, очевидно, базисная. Далее, поскольку добавление произвольного количества изолированных вершин не меняет положительную часть спектра оператора Лапласа конечного графа, получаем $S\left(G_{n}\right)=S(F)$ для всех $n \in \mathbb{N}$. Отсюда мы легко заключаем, что $S(G)=S(F)$.

Последующие несколько теорем стандартны, как и их доказательства, основанные на стандартных методах, применимых в конечном случае. Для полноты картины мы приводим однако доказательства некоторых из них.

ТЕОРема 1. Если для данного натурального числа $k$ положительное значение $\lambda_{k}(G)$ оператора Лапласа бесконечного графа $G$ существует, то оно корректно определено, т.е. не зависит от базисной последовательности $G_{n}, n \in \mathbb{N}$.

ДокАЗАТЕЛЬСтво. Пусть $G_{n}^{\prime}, G_{n}^{\prime \prime}$ - две базисные последовательности в $G$. Предположим, что $\lambda_{k}\left(G_{n_{0}}^{\prime}\right)>0$ для некоторого $n_{0} \in \mathbb{N}$, и введем обозначение $\lambda_{k}^{\prime}(G)=$ $\lim _{n \rightarrow \infty} \lambda_{k}\left(G_{n}^{\prime}\right)$. Поскольку последовательность $G_{n}^{\prime \prime}$ также является базисной в $G$, существует $n_{1} \in \mathbb{N}$ такое, что $G_{n_{0}}^{\prime} \subseteq G_{n_{1}}^{\prime \prime}$. Тогда $\lambda_{k}\left(G_{n_{1}}^{\prime \prime}\right) \geqslant \lambda_{k}\left(G_{n_{0}}^{\prime}\right)>0$, откуда $\lambda_{k}\left(G_{n_{1}}^{\prime \prime}\right)>0$. Поэтому предел $\lambda_{k}^{\prime \prime}(G)=\lim _{n \rightarrow \infty} \lambda_{k}^{\prime \prime}\left(G_{n}\right)$ также существует. Докажем, что $\lambda_{k}^{\prime}(G)=\lambda_{k}^{\prime \prime}(G)$. Для всякого $n \geqslant n_{0}$ существует число $m \geqslant n_{1}$ такое, что 
$G_{n}^{\prime} \subseteq G_{m}^{\prime \prime}$. Поэтому $\lambda_{k}\left(G_{n}^{\prime}\right) \leqslant \lambda_{k}\left(G_{m}^{\prime \prime}\right) \leqslant \lambda_{k}^{\prime \prime}(G)$. Устремляя $n \rightarrow \infty$, мы получаем $\lambda_{k}^{\prime}(G)=\lim _{n \rightarrow \infty} \lambda_{k}\left(G_{n}^{\prime}\right) \leqslant \lambda_{k}^{\prime \prime}(G)$. Поскольку по той же причине $\lambda_{k}^{\prime \prime}(G) \leqslant \lambda_{k}^{\prime}(G)$, наконец, заключаем, что $\lambda_{k}^{\prime}(G)=\lambda_{k}^{\prime \prime}(G)$.

Теорема 2. Спектр оператора Лапласа $\sigma_{L}(G)$ бесконечного графа $G$ является инвариантом графа.

Это очевидно, поскольку согласно данному выше определению спектр оператора Лапласа не зависит от нумерации множества вершин $V(G)$.

ТЕОРема 3. Пусть $H$ - конечный или бесконечный подграф бесконечного гра$\oint a G$. Тогда $|S(G)| \geqslant|S(H)|$ и для всех $k=1, \ldots,|S(H)|$ справедливо неравенство

$$
\lambda_{k}(H) \leqslant \lambda_{k}(G)
$$

ДоказАтельство. Пусть сначала $H$ - конечный подграф графа $G$, и пусть $\lambda_{k}(H)>0$ - произвольное положительное собственное значение оператора Лапласа, $k=1, \ldots,|S(H)|$. Пусть $G_{n}$ - базисная последовательность в $G$ такая, что $G_{1}=H$. Тогда $G_{1}=H \subseteq G_{n}, n \in \mathbb{N}$, откуда

$$
\lambda_{k}(H) \leqslant \lambda_{k}\left(G_{n}\right), \quad n \in \mathbb{N} .
$$

Поэтому $\lambda_{k}(G)$ существует и является положительным собственным значением оператора Лапласа для всех $k=1, \ldots,|S(H)|$. Устремляя $n \rightarrow \infty$ в последнем соотношении, получаем

$$
\lambda_{k}(H) \leqslant \lim _{n \rightarrow \infty} \lambda_{k}\left(G_{n}\right)=\lambda_{k}(G), \quad k=1, \ldots,|S(H)| .
$$

Пусть теперь $H$ - бесконечный подграф в $G$. Пусть $G_{n}$ - некоторая базисная последовательность в $G$. Тогда легко видеть, что конечные графы $H_{n}$, индуцированные из $H$ вершинами графов $G_{n}, n \in \mathbb{N}$, образуют базисную последовательность в $H$. Всякое собственное значение оператора Лапласа $\lambda_{k}(H)>0, k=1, \ldots,|S(H)|$, удовлетворяет по предположению неравенству $\lambda_{k}\left(H_{n_{0}}\right)>0$ для некоторого $n_{0} \in \mathbb{N}$. Но поскольку $H_{n} \subseteq G_{n}$, по теореме С получаем

$$
0<\lambda_{k}\left(H_{n}\right) \leqslant \lambda_{k}\left(G_{n}\right), \quad n \geqslant n_{0},
$$

т.е. $\lambda_{k}(G)$ корректно определено как положительное собственное значение оператора Лапласа графа $G$. Поэтому $|S(G)| \geqslant|S(H)|$. Теперь, устремляя $n \rightarrow \infty$ в последнем неравенстве, мы также находим, что

$$
\lambda_{k}(H)=\lim _{n \rightarrow \infty} \lambda_{k}\left(H_{n}\right) \leqslant \lim _{n \rightarrow \infty} \lambda_{k}\left(G_{n}\right)=\lambda_{k}(G)
$$

для всех $k=1, \ldots,|S(H)|$.

Из теоремы 3 вытекает, что если в бесконечном графе $G$ есть хотя бы одно ребро, т.е. если он не совпадает с пустым графом $E_{\infty}$, то $\lambda_{1}(G) \geqslant 2$. Действительно, такой граф содержит граф $K_{2}$ в качестве подграфа, а мы знаем, что $\lambda_{1}\left(K_{2}\right)=2$. 
Теорема 4. Если бесконечный граф $G$ представляет собой связную сумм графов $G_{1}, G_{2}$ (причем по крайней мере один из графов $G_{1}, G_{2}$ бесконечен), то спектр оператора Лапласа графа $G$ является подмножеством в обгединении спектров операторов Лапласа графов $G_{1}, G_{2}$ с учетом кратности нуля, т.е.

$$
\sigma_{L}\left(G_{1}+G_{2}\right) \subseteq \sigma_{L}\left(G_{1}\right) \cup \sigma_{L}\left(G_{2}\right) .
$$

ДокАЗАТЕЛЬСтво. Из определения легко вытекает, что для любых двух графов $G_{1}, G_{2}$, по крайней мере один из которых бесконечен, справедливо равенство $m_{G_{1}+G_{2}}(0)=m_{G_{1}}(0)+m_{G_{2}}(0)$; поэтому нам достаточно доказать, что $S\left(G_{1} \dot{+} G_{2}\right) \subseteq$ $S\left(G_{1}\right) \cup S\left(G_{2}\right)$. Без потери общности можно предполагать, что граф $G_{1}$ бесконечен. Пусть $G_{1, n}$ - базисная последовательность в $G_{1}$. Если граф $G_{2}$ конечен, то положим $G_{2, n}=G_{2}$ для всех $n \in \mathbb{N}$. Если же граф $G_{2}$ бесконечен, то возьмем в качестве $G_{2, n}$ базисную последовательность в $G_{2}$. В обоих случаях конечные графы $G_{n}=G_{1, n} \dot{+} G_{2, n}$ образуют базисную последовательность в $G_{1} \dot{+} G_{2}$. Выберем теперь произвольное собственное значение $\lambda \in S\left(G_{1} \dot{+} G_{2}\right)$ такое, что $\lambda=\lambda_{k}\left(G_{1} \dot{+} G_{2}\right)>0$ для некоторого $k \in \mathbb{N}$. Тогда для некоторого $n \in \mathbb{N}$ имеем $\lambda_{k}\left(G_{1, n} \dot{+} G_{2, n}\right)>0$ и

$$
\lambda_{k}\left(G_{1} \dot{+} G_{2}\right)=\lim _{n \rightarrow \infty} \lambda_{k}\left(G_{1, n} \dot{+} G_{2, n}\right) \text {. }
$$

Поскольку графы $G_{1, n}, G_{2, n}$ конечны, мы заключаем, что $S\left(G_{1, n} \dot{+} G_{2, n}\right)=S\left(G_{1, n}\right) \cup$ $S\left(G_{2, n}\right)$, откуда

$$
\lambda_{k}\left(G_{1, n} \dot{+} G_{2, n}\right)=\lambda_{i(k, n)}\left(G_{\varepsilon_{n}, n}\right),
$$

где $\varepsilon_{n} \in\{1,2\}$ и $i(k, n) \leqslant k$. Поскольку это равенство выполняется для всех $n=1,2, \ldots$, очевидно, существует подпоследовательность $n_{1}<n_{2}<\cdots$ такая, что $\varepsilon_{n_{p}}=$ const $=\varepsilon \in\{1,2\}$ и $i\left(k, n_{p}\right)=$ const $=m \leqslant k, p=1,2, \ldots$. Для такой подпоследовательности имеем

$$
\lambda_{k}\left(G_{1, n_{p}} \dot{+} G_{2, n_{p}}\right)=\lambda_{m}\left(G_{\varepsilon, n_{p}}\right), \quad p=1,2, \ldots .
$$

Заметив, что $G_{1, n_{p}}, G_{2, n_{p}}$ и $G_{1, n_{p}} \dot{+} G_{2, n_{p}}$ также являются базисными последовательностями в графах $G_{1}, G_{2}, G_{1}+G_{2}$ соответственно и устремляя $p \rightarrow \infty$ в последнем соотношении, мы заключаем, что

$$
\lambda_{k}\left(G_{1} \dot{+} G_{2}\right)=\lim _{p \rightarrow \infty} \lambda_{k}\left(G_{1, n_{p}} \dot{+} G_{2, n_{p}}\right)=\lim _{p \rightarrow \infty} \lambda_{m}\left(G_{\varepsilon, n_{p}}\right)=\lambda_{m}\left(G_{\varepsilon}\right) \in S\left(G_{\varepsilon}\right),
$$

где $\varepsilon \in\{1,2\}$, что и доказывает включение $S\left(G_{1} \dot{+} G_{2}\right) \subseteq S\left(G_{1}\right) \cup S\left(G_{2}\right)$.

Ниже мы приведем несколько примеров, в которых $S\left(G_{1} \dot{+} G_{2}\right) \neq S\left(G_{1}\right) \cup S\left(G_{2}\right)$. Индукцией по $m \in \mathbb{N}, m \geqslant 2$, можно доказать и более общую теорему.

ТеОрема 5. Пусть $G_{1}, \ldots, G_{m}, m \geqslant 2,-$ конечные или бесконечные графы, хотя бы один из которых бесконечен. Тогда

$$
S\left(G_{1} \dot{+} \cdots \dot{+} G_{m}\right) \subseteq S\left(G_{1}\right) \cup \cdots \cup S\left(G_{m}\right) .
$$

Отметим, что теорема 5 перестает быть верной в случае, когда бесконечный граф $G$ является прямой суммой бесконечного набора слагаемых $G_{1}, G_{2}, \ldots$. Например, если $G=K_{2} \dot{+} K_{3} \dot{+} K_{4} \dot{+} \cdots$, то конечный граф $K_{n}$ является подграфом в $G$ для любого $n \geqslant 2$, откуда по теореме 3 легко видеть, что $S(G)=\{\infty, \infty, \ldots\}$. Однако это множество не является подмножеством в $\bigcup_{n=1}^{\infty} S\left(K_{n+1}\right)$. 


\section{3. Примеры.}

ПримеР 1. Для бесконечного графа $E_{\infty}$, состоящего лишь из изолированных вершин, имеем $\sigma_{L}\left(E_{\infty}\right)=\{; 0,0, \ldots\}$ при кратности нуля $m(0)=\infty$. Также легко видеть, что если у бесконечного графа $G$ нет положительных собственных значений оператора Лапласа, то он изоморфен графу $E_{\infty}$.

ПримеР 2. Для полного бесконечного графа $K_{\infty}$ имеем $\sigma_{L}\left(K_{\infty}\right)=\{\infty, \infty, \ldots ; 0\}$ с кратностью нуля $m(0)=1$. Это очевидно, поскольку полные графы $K_{n}$ на $n$ вершинах, $n \in \mathbb{N}$, образуют базисную последовательность графа $K_{\infty}$, а

$$
\sigma_{L}\left(K_{n}\right)=\{\underbrace{n, \ldots, n}_{n-1} ; 0\} .
$$

Пример 3. Для бесконечной в одну сторону цепочки $P_{\infty}^{+}$и бесконечной в обе стороны цепочки $P_{\infty}$ имеем $\sigma_{L}\left(P_{\infty}^{+}\right)=\sigma_{L}\left(P_{\infty}\right)=\{4,4, \ldots ; 0\}$ при кратности нуля $m(0)=1$ в обоих случаях. Действительно, в обоих случаях пути $P_{n}$ на $n$ вершинах, $n \in \mathbb{N}$, образуют базисную последовательность подграфов и, как известно,

$$
\lambda_{k}\left(P_{n}\right)=2\left(1+\cos \frac{\pi k}{n}\right), \quad k=1, \ldots, n-1,
$$

а $\lambda_{n}\left(P_{n}\right)=0$. Поскольку для данного $k \in \mathbb{N}$ имеем $\lambda_{k}\left(P_{n}\right) \rightarrow 4$ при $n \rightarrow \infty$, мы заключаем, что $\lambda_{k}\left(P_{\infty}^{+}\right)=\lambda_{k}\left(P_{\infty}\right)=4, k=1,2, \ldots$.

ПримеР 4. Для бесконечной звезды $K(1, \infty)$, т.е. для полного двудольного графа с долями $A=\{1\}$ и $B=\mathbb{N}=\{1,2, \ldots\}$, имеем $\sigma_{L}(K(1, \infty))=\{\infty, 1,1,1, \ldots ; 0\}$. Действительно, графы $K(1, n), n \in \mathbb{N}$, образуют базисную последовательность подграфов в $K(1, \infty)$ и, как хорошо известно,

$$
\sigma_{L}(K(1, n))=\{n+1, \underbrace{1,1, \ldots, 1}_{n-1}, 0\} .
$$

Пример 5. Аналогично, для данного числа $p \in \mathbb{N}$ обозначим через $K(p, \infty)$ полный двудольный граф с долями $A=\{1,2, \ldots, p\}$ и $B=\mathbb{N}$. Тогда

$$
\sigma_{L}(K(p, \infty))=\{\underbrace{\infty, \ldots, \infty}_{p}, p, p, \ldots ; 0\}
$$

с кратностью собственного значения $\infty$, равной $p$. Действительно, достаточно заметить, что последовательность графов $K(p, n), n \in \mathbb{N}$, является базисной в рассматриваемом графе и, как хорошо известно,

$$
S(K(p, n))=\{p+n, \underbrace{n, n, \ldots, n}_{p-1}, \underbrace{p, p, \ldots, p}_{n-1}\} .
$$

ПримеР 6. Пусть $K(\infty, \infty)$ - полный двудольный граф, обе части которого бесконечны. Тогда $\sigma_{L}(K(\infty, \infty))=\{\infty, \infty, \ldots ; 0\}$. Действительно, в этом случае графы $K(n, n), n \in \mathbb{N}$, образуют базисную последовательность в рассматриваемом графе и, как хорошо известно,

$$
S(K(n, n))=\{2 n, \underbrace{n, n, \ldots, n}_{2 n-2}\} .
$$


ПримеР 7. Если $G_{1}=P_{\infty}$ и $G_{2}=K_{2}$, то $S\left(G_{1}\right)=\{4,4, \ldots\}, S\left(G_{2}\right)=\{2\}$, $S\left(G_{1} \dot{+} G_{2}\right)=\{4,4, \ldots\}=S\left(G_{1}\right) \neq S\left(G_{1}\right) \cup S\left(G_{2}\right)$. Аналогично, если для любого числа $m \geqslant 2$ мы положим $G_{1}=P_{\infty}, G_{2}=\cdots=G_{m}=K_{2}$, то $S\left(G_{2}\right)=\cdots=$ $S\left(G_{m}\right)=\{2\}$ и

$$
S\left(G_{1}+G_{2} \dot{+} \cdots \dot{+} G_{m}\right)=S\left(G_{1}\right)=\{4,4, \ldots\} \neq S\left(G_{1}\right) \cup\{\underbrace{2, \ldots, 2}_{m-1}\}=\bigcup_{i=1}^{m} S\left(G_{i}\right) .
$$

4. Мощность множества $S(G)$. Легко видеть, что если в бесконечном графе $G$ есть хотя бы одно ребро, то спектр оператора Лапласа $\sigma_{L}(G)$ содержит по крайней мере одно положительное собственное значение, т.е. его положительная часть $S(G)$ всегда непуста. Конечные или бесконечные графы с непустой положительной частью называются нетривиалъными. Сформулируем одно свойство связных бесконечных графов.

ТеОрема 6. Если $G$ - связный бесконечный граф или хотя бы одна из его компонент связности бесконечна, то в спектре его оператора Лапласа бесконечно много положительных элементов.

ДокАЗАТЕЛЬСтво. Предположим сначала, что граф $G$ связен и бесконечен. Пусть $G_{n}, n \in \mathbb{N},-$ произвольная базисная последовательность графа $G$, целиком состоящая из конечных графов. Тогда для каждого $k \in \mathbb{N}$ справедлива оценка $\lambda_{k}\left(G_{n}\right)>0$, если $\left|G_{n}\right| \geqslant k+1$, т.е. если $n$ достаточно велико. Мы заключаем, что $\lambda_{k}(G)>0$ для всякого $k \in \mathbb{N}$. Поэтому если граф $G$ несвязен, но в нем есть бесконечная связная компонента $C$, то в спектре оператора Лапласа графа $C$ бесконечно много положительных элементов. Граф $C$ является подграфом в $G$, откуда немедленно вытекает, что то же самое утверждение справедливо и для всего графа $G$.

В следующем утверждении исследуется ситуация, когда у бесконечного графа в точности $p, p \in \mathbb{N}$, положительных собственных значений оператора Лапласа.

Теорема 7. Спектр оператора Лапласа $\sigma_{L}(G)$ бесконечного графа $G$ содержит в точности $p, p \in \mathbb{N}$, положительных собственных значений (с учетом кратностей), если и только если существует такое целое число $r \leqslant p$ и такой конечный граф $F$ порядка $p+r$, имеющий в точности $r$ нетривиалъных компонент связноcmu, чmо $G=F+E_{\infty}$.

Ясно, что в таком случае все эти собственные значения конечны.

ДокАЗАТЕЛЬСтво. Предположим сначала, что $G=F \dot{+} E_{\infty}$, где граф $F$ конечен. Поскольку по предложению $1 S(G)=S(F)$, мы заключаем, что положительные собственные значения оператора Лапласа графа $G$ в точности совпадают с положительными собственными значениями оператора Лапласа графа $F$, а значит, их число конечно. Хорошо известно, что $|S(F)|=|F|-m_{F}(0)=(p+r)-r=p$; поэтому у графа $G$ в точности $p$ положительных собственных значений оператора Лапласа. Наоборот, предположим, что $G$ - бесконечный граф со свойством $\lambda_{1}(G) \geqslant \cdots \geqslant \lambda_{p}(G)>0$ и $\lambda_{p+1}(G)=0$. Тогда $\lambda_{p+1}(H)=0$ для всякого конечного подграфа $H \subseteq G$ порядка 
$n>p+1$. Если одна из связных компонент $G_{1}$ графа $G$ бесконечна, то у нее бесконечно много положительных собственных значений оператора Лапласа и по теореме 3 то же самое справедливо и для всего графа $G$; приходим к противоречию. Поэтому все компоненты связности графа $G$ должны быть конечными. Пусть $G_{1}, \ldots, G_{s}-$ произвольные нетривиальные компоненты связности графа $G$. Поскольку число положительных собственных значений оператора Лапласа индуцированного подграфа $H=G_{1} \dot{+} \cdots \dot{+} G_{s}$ графа $G$ не превосходит $p$, мы заключаем, что $\left|G_{1}\right|+\cdots \dot{+}\left|G_{s}\right|-s \leqslant p$. Но из неравенств $\left|G_{1}\right|, \ldots,\left|G_{s}\right| \geqslant 2$ легко вытекает, что $s \leqslant p$. Пусть, наконец, $r-$ число всех нетривиальных компонент связности графа $G, 1 \leqslant r \leqslant p$. Обозначив эти компоненты через $G_{1}, \ldots, G_{r}$, получаем $G=G_{1} \dot{+} \cdots \dot{+} G_{r} \dot{+} E_{\infty}$. Поэтому, полагая $F=G_{1} \dot{+} \cdots \dot{+} G_{r}$, имеем $G=F \dot{+} E_{\infty}$. Из того, что число положительных собственных значений оператора Лапласа графа $G$ в точности такое же, как у $F$, легко вытекает теперь, что порядок графа $F$ равен $p+r$. Теорема доказана.

Из последней теоремы вытекает, что если положительная часть спектра оператора Лапласа $S(G)$ бесконечного графа $G$ конечна, то $G$ представляет собой бесконечное расширение некоторого конечного графа. В этом случае кратность нуля $m_{G}(0)=\infty$.

Из теорем 6 и 7 легко вытекает такое следствие.

СледствиЕ 1. (а) Число положительных собственных значений в спектре оператора Лапласа бесконечного графа $G$ бесконечно, если и только если либо $G$ состоит из конечного числа компонент связности, либо среди его компонент связности бесконечно много нетривиальных.

(b) Если в графе $G$ нет изолированньх вершин, то положительная часть $S(G)$ спектра его оператора Лапласа бесконечна.

Пусть теперь $G$ - произвольный бесконечный граф с бесконечно большим числом положительных собственных значений в спектре оператора Лапласа. Обозначим эти значения через $\lambda_{1}(G) \geqslant \lambda_{2}(G) \geqslant \cdots>0$. Очевидно, существует предел

$$
\lambda(G)=\lim _{n \rightarrow \infty} \lambda_{n}(G)
$$

причем $0 \leqslant \lambda(G) \leqslant \infty$. Этот предел, очевидно, является инвариантом графа. Мы хотим показать, что для любого бесконечного графа $G$ без изолированных вершин справедлива оценка $\lambda(G) \geqslant 1$. Она позволяет нам назвать по (формальной) аналогии с конечным случаем величину $\lambda(G)$ алгебраической связностью бесконечного графа.

5. Нижние оценки для собственных значений оператора Лапласа. В этом пункте мы доказываем одно характеристическое свойство спектра оператора Лапласа для связных бесконечных графов. Обозначим через $\operatorname{diam}(G)$ диаметр связного бесконечного графа $G$ (который является либо конечным натуральным числом $n$, либо бесконечным порядковым числом $\aleph_{0}$, или, короче, $\left.\infty\right)$. Очевидно, что либо $\operatorname{diam}(G)<\infty$, либо $\operatorname{diam}(G)=\infty$. 
Tеорема 8. Пусть $G$ - связный бесконечный граф. Если $\operatorname{diam}(G)<\infty$, то $\lambda_{1}(G)=\infty$ и $\lambda_{k}(G) \geqslant 1$ для всех $k \geqslant 2$. Если $\operatorname{diam}(G)=\infty$, mо $\lambda_{k}(G) \geqslant 4$ для всех $k \in \mathbb{N}$.

ДокАзАтельство. Предположим сначала, что $\operatorname{diam}(G)<\infty$. Тогда легко видеть, что $G$ содержит бесконечную звезду $H=K(1, \infty)$ в качестве подграфа. Поскольку, как мы уже видели, $\lambda_{1}(H)=\infty$ и $\lambda_{k}(H)=1$ для $k \geqslant 2$, мы немедленно заключаем, что $\lambda_{1}(G)=\infty$ и $\lambda_{k}(G) \geqslant 1, k \geqslant 2$. Теперь предположим, что $\operatorname{diam}(G)=\infty$. Тогда $G$ содержит индуцированные подграфы, образующие последовательность цепочек $P_{n_{i}}, n_{1}<n_{2}<\cdots$. Это означает, что $\lambda_{k}(G) \geqslant \lambda_{k}\left(P_{n_{i}}\right)$ для всех $k \in \mathbb{N}$ и, устремляя $n_{i} \rightarrow \infty$, мы немедленно заключаем, что $\lambda_{k}(G) \geqslant 4, k \in \mathbb{N}$.

Из последней теоремы немедленно вытекает

СлЕДСТВИЕ 2. Если $G$ - произволъный связный бесконечный граф или бесконечный граф, хотя бы одна из компонент связности которого бесконечна, то $\lambda_{1}(G) \geqslant 4, \lambda_{k}(G) \geqslant 1$ для всех $k \geqslant 2$, откуда $\lambda(G) \geqslant 1$.

Легко видеть, что для связных бесконечных графов инвариант $\lambda(G)$ достигает обоих крайних значений 1 и $\infty$. Например, $\lambda(K(1, \infty))=1$ и $\lambda\left(K_{\infty}\right)=\infty$.

6. $\infty$ как собственное значение оператора Лапласа. Мы уже видели на примерах, что есть такие бесконечные графы, все собственные значения оператора Лапласа которых конечны, есть бесконечные графы, имеющие как конечные, так и бесконечные собственные значения, и есть бесконечные графы, все собственные значения оператора Лапласа которых бесконечны. В дальнейшем мы даем полное описание необходимых и достаточных условий, при которых реализуется каждая из этих возможностей. Как и следовало ожидать, этот вопрос тесно связан со свойствами последовательности валентностей вершин рассматриваемого бесконечного графа. Пусть $G$ - бесконечный граф, и пусть $d_{n}$ обозначает валентность вершины $v_{n}$, которая может быть либо конечным числом $0,1,2, \ldots$, либо $\aleph_{0}$, для краткости $\infty$. В частности, если $G=E_{\infty}$, то $d_{1}=d_{2}=\cdots=0$, а для $G=K_{\infty}$ имеем $d_{1}=d_{2}=\cdots=\infty$. И здесь мы не накладываем никаких ограничений на валентности $d_{n}, n \in \mathbb{N}$, в частности, не требуем, чтобы валентности были конечны и ограничены.

Теорема 9. Все собственные значения в спектре оператора Лапласа $\sigma_{L}(G)$ бесконечного графа $G$ конечны, т.е. $\lambda_{1}(G)<\infty$, в том и только том случае, если последовательность валентностей $d_{n}$ ограничена некоторым числом $d<\infty$. $B$ этом случае введем обозначение $d=\max \left\{d_{n} \mid n \in \mathbb{N}\right\} ;$ если $G$ не является пустым графом $E_{\infty}$, mо

$$
d+1 \leqslant \lambda_{1}(G) \leqslant 2 d
$$

ДокАзАтЕЛЬство. Для графа $G=E_{\infty}$ теорема, очевидно, верна. Предположим теперь, что $G \neq E_{\infty}$ и $d=\max \left\{d_{1}, d_{2}, \ldots\right\}-$ конечное число. Пусть $G_{n}, n \in \mathbb{N},-$ произвольная базисная последовательность подграфов в $G$. Поскольку валентности вершин в графах $G_{n}$ не превосходят валентностей тех же вершин в $G$, справедлива 
оценка

$$
\lambda_{1}\left(G_{n}\right) \leqslant 2 d\left(G_{n}\right) \leqslant 2 d,
$$

где через $d\left(G_{n}\right)$ обозначена максимальная валентность в графе $G_{n}$. Поэтому

$$
\lambda_{1}(G)=\lim _{n \rightarrow \infty} \lambda_{1}\left(G_{n}\right) \leqslant 2 d<\infty .
$$

Наоборот, предположим, что последовательность валентностей $d_{n}, n \in \mathbb{N}$, бесконечного графа $G$ неограниченна. Если существует вершина $v \in V(G)$, для которой $d(v)=\infty$, то $G$ содержит бесконечную звезду $H=K(1, \infty)$ в качестве подграфа. В этом случае имеем $\lambda_{1}(G) \geqslant \lambda_{1}(H)=\infty$, откуда $\lambda_{1}(G)=\infty$. Если для всякой вершины $v \in V(G)$ справедливо неравенство $d(v)<\infty$, то имеется последовательность $d_{n_{1}}<d_{n_{2}}<\cdots$ конечных валентностей вершин в $G$ такая, что $d_{n_{i}} \rightarrow \infty$ при $i \rightarrow \infty$. Тогда для всякого $i \in \mathbb{N}$ существует конечный граф $H_{i} \subseteq G$, максимальная валентность вершины в котором равна $d_{n_{i}}$. Поскольку $\lambda_{1}(G) \geqslant \lambda_{1}\left(H_{i}\right) \geqslant d_{n_{i}}+1$, имеем $\lambda_{1}(G)=\infty$. Для доказательства левого неравенства в (3) обозначим через $H$ конечный граф - индуцированный подграф в $G,-$ максимальная валентность вершины в котором (в $H$ ) в точности равна $d$. Тогда в силу соответствующего свойства конечных графов имеем $\lambda_{1}(G) \geqslant \lambda_{1}(H) \geqslant d+1$.

Из теоремы 9 немедленно вытекает

СЛЕДСТВИЕ 3. Максималъное собственное значение в спектре оператора Лапласа $\sigma_{L}(G)$ бесконечного графа $G$ равно $\lambda_{1}(G)=\infty$ в том и только том случае, если соответствующая последовательность валентностей вериин неограниченна.

Условие следствия 3 означает, очевидно, что либо степень некоторой вершины в $G$ бесконечна, либо существует последовательность вершин, валентности которых конечны и строго возрастают: $d_{n_{1}}<d_{n_{2}}<\cdots$.

Лемма 1. Если $G_{1}, G_{2}-$ два бесконечных графа такие, что $V\left(G_{1}\right) \cap V\left(G_{2}\right)=\varnothing$ и $m_{G_{1}}(\infty) \geqslant p_{1}, m_{G_{2}}(\infty) \geqslant p_{2}$ для некоторых натуралъных чисел $p_{1} u p_{2}$, то $m_{G_{1}+G_{2}}(\infty) \geqslant p_{1}+p_{2}$.

ДокАЗАтЕЛьство. Пусть $n \mapsto G_{i, n}, n \in \mathbb{N},-$ базисная последовательность в графе $G_{i}, i=1,2$. Тогда, очевидно, $G_{n}=G_{1, n} \dot{+} G_{2, n}, n \in \mathbb{N},-$ базисная последовательность в $G_{1}+G_{2}$. Пусть $M>0$ - произвольное положительное число. Поскольку согласно предположению $m_{G_{1}}(\infty) \geqslant p_{1}$ и $m_{G_{2}}(\infty) \geqslant p_{2}$, существует натуральное число $n_{0}=n_{0}(M)$ такое, что для всякого $n \geqslant n_{0}$ справедливы неравенства $\lambda_{p_{1}}\left(G_{1, n}\right) \geqslant M$ и $\lambda_{p_{2}}\left(G_{2, n}\right) \geqslant M$. Поскольку $S\left(G_{1, n} \dot{+} G_{2, n}\right)=S\left(G_{1, n}\right) \cup S\left(G_{2, n}\right)$, мы заключаем, что $\lambda_{p_{1}+p_{2}}\left(G_{1, n} \dot{+} G_{2, n}\right) \geqslant M, n \geqslant n_{0}$, и поэтому $\lambda_{p_{1}+p_{2}}\left(G_{1} \dot{+} G_{2}\right) \geqslant M$. Отсюда вытекает, что $\lambda_{p_{1}+p_{2}}\left(G_{1} \dot{+} G_{2}\right)=\infty$ и, значит, $m_{G_{1}+G_{2}}(\infty) \geqslant p_{1}+p_{2}$.

Теорема 10. Если для некоторого натуралъного числа $p \in \mathbb{N}$ в $G$ есть по крайней мере $p$ вершин бесконечной валентности, то $\lambda_{1}(G)=\cdots=\lambda_{p}(G)=\infty u$ в спектре оператора Лапласа графа $G$ есть по крайней мере р бесконечных собственных значений.

ДокАзАтЕльство. Мы докажем общее утверждение индукцией по $p$. По теореме 9 оно справедливо при $p=1$. Предположим теперь, что $p \geqslant 2$ и что теорема 
верна для всякого целого числа $q<p$; докажем ее для $q=p$. Без потери общности можно предположить, что $d_{1}=\cdots=d_{p}=\infty$. Обозначим через $A_{0}$ подграф в $G$, индуцированный вершинами $v_{1}, \ldots, v_{p}$, а через $A_{i}, i=1, \ldots, p,-$ подграф графа $G$, индуцированный всеми соседями вершины $v_{i}$ в $G$, не лежащими в $A_{0}$. Ясно, что все графы $A_{i} \subseteq G$ бесконечны. Удалим из каждого из подграфов $A_{0}, A_{1}, \ldots, A_{p}$ все его ребра, а также все ребра, соединяющие подграфы $A_{i}$ и $A_{j}, 1 \leqslant i<j \leqslant p$. Обозначим граф с множеством вершин $V\left(A_{0}\right) \cup V\left(A_{1}\right) \cup \cdots \cup V\left(A_{p}\right)$, полученный таким образом, через $H$. Очевидно, что этот граф бесконечен и является подграфом в рассматриваемом графе $G$. Рассмотрим теперь множество вершин $V\left(A_{1}\right)$. Очевидно, что оно представляет собой несвязное объединение $2^{p-1}$ подмножеств вида $V\left(A_{1}\right) \cap M_{2} \cap \cdots \cap M_{p}$, где либо $M_{i}=V\left(A_{i}\right)$, либо $M_{i}=V\left(A_{i}\right)^{c}$ (дополнение к $\left.V\left(A_{i}\right)\right)$, $i=2, \ldots, p$. Поскольку множество вершин $V\left(A_{1}\right)$ бесконечно, по крайней мере одно из этих подмножеств также бесконечно. Предположим сначала, что это подмножество $B=V\left(A_{1}\right) \cap \cdots \cap V\left(A_{p}\right)$. Тогда, очевидно, $G$ содержит в качестве подграфа полный двудольный граф $K(p, \infty)$ с долями $A_{0}$ и $B$. Поскольку у такого графа по крайней мере $p$ бесконечных собственных значений оператора Лапласа, мы немедленно выводим то же свойство и для всего графа $G$. Теперь мы можем без потери общности предположить, что множество

$$
B=V\left(A_{1}\right) \cap V\left(A_{2}\right) \cap \cdots \cap V\left(A_{q}\right) \cap V\left(A_{q+1}\right)^{c} \cap \cdots \cap V\left(A_{p}\right)^{c}
$$

бесконечно для некоторого $q, 1 \leqslant q<p$. Тогда подграф $H_{1}$ графа $G$, индуцированный вершинами $\left\{v_{1}, \ldots, v_{q}\right\}$ и $B$, является полным двудольным графом вида $K(q, \infty)$ и $m_{H_{1}}(\infty) \geqslant q$. С другой стороны, пусть $H_{2}$ - подграф в $G$, индуцированный вершинами $v_{q+1}, \ldots, v_{p}$ и подмножествами $V\left(A_{q+1}\right), \ldots, V\left(A_{p}\right)$. Применяя предположение индукции к подграфу $H_{2}$, мы заключаем, что $m_{H_{2}}(\infty) \geqslant p-q$. Поэтому по лемме 1 у графа $H_{1} \dot{+} H_{2} \subseteq G$ по крайней мере $p$ бесконечных собственных значений спектра оператора Лапласа, а значит, и у графа $G$ спектр содержит по крайней мере $p$ бесконечных собственных значений.

Из этой теоремы немедленно вытекает

СлеДСТвИЕ 4. Если $G$ - бесконечный граф с бесконечно болъшим количеством вершин степени $d_{n_{i}}=\infty, n_{1}<n_{2}<\cdots$, mо $S(G)=\{\infty, \infty, \ldots\}$.

Теорема 11. Если $G$ - бесконечный граф, в котором есть последовательность вершин конечных валентностей $d_{n_{1}}<d_{n_{2}}<\cdots$ (которые стремятся $\kappa$ бесконечности), $\operatorname{mo~} S(G)=\{\infty, \infty, \ldots\}$.

Мы опускаем доказательство, поскольку оно проводится с помощью техники, аналогичной использованной в доказательстве теоремы 10.

Теорема 12. Если в бесконечном графе $G$ в точности $p$ вершин бесконечной валентности, а валентности всех остальных вершин ограничены некоторым целым числом $d$, то спектр оператора Лапласа графа $G$ содержст в точности $p$ бесконечных собственных значений.

ДоКАЗАТЕЛЬСтво. Без потери общности мы можем предполагать, что $d_{1}=\cdots=$ $d_{p}=\infty$, а для всех остальных вершин $d_{n} \leqslant d, n>p$. Теорема 10 утверждает, что у 
оператора Лапласа графа $G$ по крайней мере $p$ бесконечных собственных значений, поэтому нам осталось только доказать, что все остальные собственные значения конечны. Мы докажем больше; именно, что все остальные критические значения ограничены общей константой. Обозначим через $\widetilde{G}$ бесконечный граф с тем же множеством вершин $V(G)$, полученный из $G$ соединением всех вершин $v_{1}, \ldots, v_{p}$ между собой и со всеми вершинами графа $G$. Очевидно, что $G$ является подграфом в $\widetilde{G}$. Теперь мы собираемся доказать, что $\lambda_{p+1}(\widetilde{G}) \leqslant 2 d+p$ и, следовательно, $\lambda_{p+1}(G) \leqslant 2 d+p$. Обозначим через $K_{p}$ подграф в $\widetilde{G}$, индуцированный вершинами $v_{1}, \ldots, v_{p}$ (полный граф на $p$ вершинах), и пусть $H_{n} \subseteq \widetilde{G} \backslash K_{p}$ - базисная последовательность подграфов в графе $G_{0}=\widetilde{G} \backslash K_{p}$. Тогда, очевидно, подграфы $G_{n}=K_{p} \nabla H_{n}$, $n \in \mathbb{N}$, образуют базисную последовательность подграфов в $\widetilde{G}$. Полагая $\left|H_{n}\right|=m_{n}$, $n \in \mathbb{N}$, и переходя к дополнительным графам, получаем

$$
\begin{aligned}
\lambda_{p+1}\left(G_{n}\right) & =\lambda_{p+1}\left(K_{p} \nabla H_{n}\right)=m_{n}+p-\lambda_{m_{n}-1}\left(\overline{K_{p} \nabla H_{n}}\right)=m_{n}+p-\lambda_{m_{n}-1}\left(E_{p}+\overline{H_{n}}\right) \\
& =m_{n}+p-\lambda_{m_{n}-1}\left(\overline{H_{n}}\right)=p+\lambda_{1}\left(H_{n}\right) .
\end{aligned}
$$

Поскольку $\lambda_{1}\left(H_{n}\right) \leqslant 2 d$, мы в конце концов получаем $\lambda_{p+1}\left(G_{n}\right) \leqslant p+2 d$. Поэтому $\lambda_{p+1}(G) \leqslant 2 d+p$ и, значит, $\lambda_{n}(G) \leqslant 2 d+p$ для всех $n \geqslant p+1$.

7. Спектр оператора Лапласа дополнительного графа. Теперь обсудим соотношение между спектром оператора Лапласа бесконечного графа $G$ и дополнительного к нему графа $\bar{G}$.

ПРЕДЛОЖЕНИЕ 2. Если по крайней мере одно собственное значение оператора Лапласа бесконечного графа $G$ конечно и положительно или у $G$ нет положительных собственных значений оператора Лапласа, то $S(\bar{G})=\{\infty, \infty, \ldots\}$.

ДокАзАтельство. Во-первых, если в спектре оператора Лапласа графа $G$ нет положительных собственных значений, то, как мы знаем, $G$ изоморфен графу $E_{\infty}$. Дополнительный к нему подграф $\bar{G}$ изоморфен полному бесконечному графу $K_{\infty}$, спектр оператора Лапласа которого содержит бесконечно много значений $\infty$. Предположим теперь, что у оператора Лапласа на $G$ есть по крайней мере одно конечное положительное собственное значение $\lambda_{k}(G)$. Обозначим валентности вершин графов $G$ и $\bar{G}$ через $d_{1}, d_{2}, \ldots$ и $\bar{d}_{1}, \bar{d}_{2}, \ldots$ соответственно. Поскольку $\lambda_{k}(G)<\infty$ для некоторого $k \in \mathbb{N}$, мы можем предполагать, что $d_{1}=\cdots=d_{p}=\infty$ для некоторого $p, 0 \leqslant p<k$, и $d_{n} \leqslant d<\infty$ для всех $n \geqslant p+1$. Отсюда мы заключаем, что $\bar{d}_{n}=\infty$ для всех $n \geqslant p+1$, и в силу следствия 4 находим $S(\bar{G})=\{\infty, \infty, \ldots\}$.

ПрЕДЛОЖЕНИЕ 3. Если положительная часть $S(G)$ спектра оператора Лапласа бесконечного графа $G$ (без изолированных вериин) состоит лишь из значений $\infty$, то любая возможная кратность собственного значения $\infty$ в положительном спектре оператора Лапласа $S(\bar{G})$ дополнительного графа реализуема: $m_{\bar{G}}(\infty)=0$, $m_{\bar{G}}(\infty) \in \mathbb{N} u m_{\bar{G}}(\infty)=\infty$.

ДокАзАтельство. Разумеется, доказательство мы проведем путем предъявления соответствующих примеров. Во-первых, если существует такое натуральное число $d$, что $d_{n}=\infty$ и $\bar{d}_{n} \leqslant d$ для всех $n \in \mathbb{N}$, то, очевидно, $S(G)=\{\infty, \infty, \ldots\}$ и 
$\infty \notin S(\bar{G})$. Далее, если $d_{n}=\bar{d}_{n}=\infty$ для всех $n \in \mathbb{N}$, то, очевидно, $S(G)=S(\bar{G})=$ $\{\infty, \infty, \ldots\}$. Наконец, если для некоторых натуральных чисел $p$ и $d, d_{k}=\bar{d}_{k}=\infty$, $k=1,2, \ldots, p$, и $d_{n}=\infty$ и $\bar{d}_{n} \leqslant d$ для всех $n>p$, то $S(G)=\{\infty, \infty, \ldots\}$ и $m_{\bar{G}}(\infty)=p$.

\section{СПИСОК ЦИТИРОВАННОЙ ЛИТЕРАТУРЫ}

[1] B. Mohar, "The spectrum of an infinite graph", Linear Algebra Appl., 48 (1982), 245-256.

[2] B. Mohar, W. Woess, "A survey on spectra of infinite graphs", Bull. London Math. Soc., 21 (1989), 209-234.

[3] B. Mohar, "The Laplacian spectrum of graphs", Graph Theory, Combinatorics and Applications, eds. Y. Alard, G. Chartrand, O. R. Ollerman, A. J. Schwenk, J. Willy, New York, 1991, 871-898.

[4] B. Mohar, "Some relations between analytic and geometric properties of infinite graphs", Discrete Math., 95 (1991), 193-219.

[5] A. Torgašev, "Infinite graphs with the least limiting eigenvalue greater than -2 ", Linear Algebra Appl., 82 (1986), 133-141.

[6] A. Torgašev, "The limiting spectrum of infinite graphs", Review of Research Novi Sad, $\mathbf{2 3}$ (1993), 259-268.

[7] D. M. Cvetković, M. Doob, I. Gutman, A. Torgašev, Recent Results in the Theory of Graph Spectra, Ann. Discrete Math., 36, North-Holland, Amsterdam, 1988.

[8] D. M. Cvetković, M. Doob, H. Sachs, Spectra of Graphs, Academic Press, 1979.

[9] R. Grone, R. Merris, V.S. Sunder, "The Laplacian spectrum of a graph", SIAM J. Matrix Anal. Appl., 11 (1990), 218-238.

[10] R. Merris, "Laplacian matrices of graphs, A survey", Linear Algebra Appl., 197-198 (1994), 143-176.

[11] A. Torgašev, "On spectra of infinite graphs", Publ. Inst. Math. (Beograd), 29 (43) (1981), 269-282.

[12] A. Torgašev, "On infinite graphs whose spectrum is greater than -2", Bull. Acad. Serbe Sci. Arts 84 (Sci. Math.), 13 (1984), 21-35.

\section{А. Торгашев}

11000 Beograd, Serbia and Montenegro

E-mail: torgasev@matf.bg.ac.yu

\section{М. Петрович}

Faculty of Science, Radoja Domanovića 12, 34000 Kragujevac, Serbia and Montenegro

E-mail: petrovic@knez.uis.kg.ac.yu 